This item was submitted to Loughborough's Research Repository by the author.

Items in Figshare are protected by copyright, with all rights reserved, unless otherwise indicated.

\title{
Frost heave in colloidal soils
}

PLEASE CITE THE PUBLISHED VERSION

http://dx.doi.org/10.1137/100788197

PUBLISHER

(c) Society for Industrial and Applied Mathematics

VERSION

VoR (Version of Record)

LICENCE

CC BY-NC-ND 4.0

REPOSITORY RECORD

Peppin, Stephen, Apala Majumdar, Robert Style, and Graham C. Sander. 2019. "Frost Heave in Colloidal Soils". figshare. https://hdl.handle.net/2134/11066. 
This item was submitted to Loughborough's Institutional Repository (https://dspace.lboro.ac.uk/) by the author and is made available under the following Creative Commons Licence conditions.

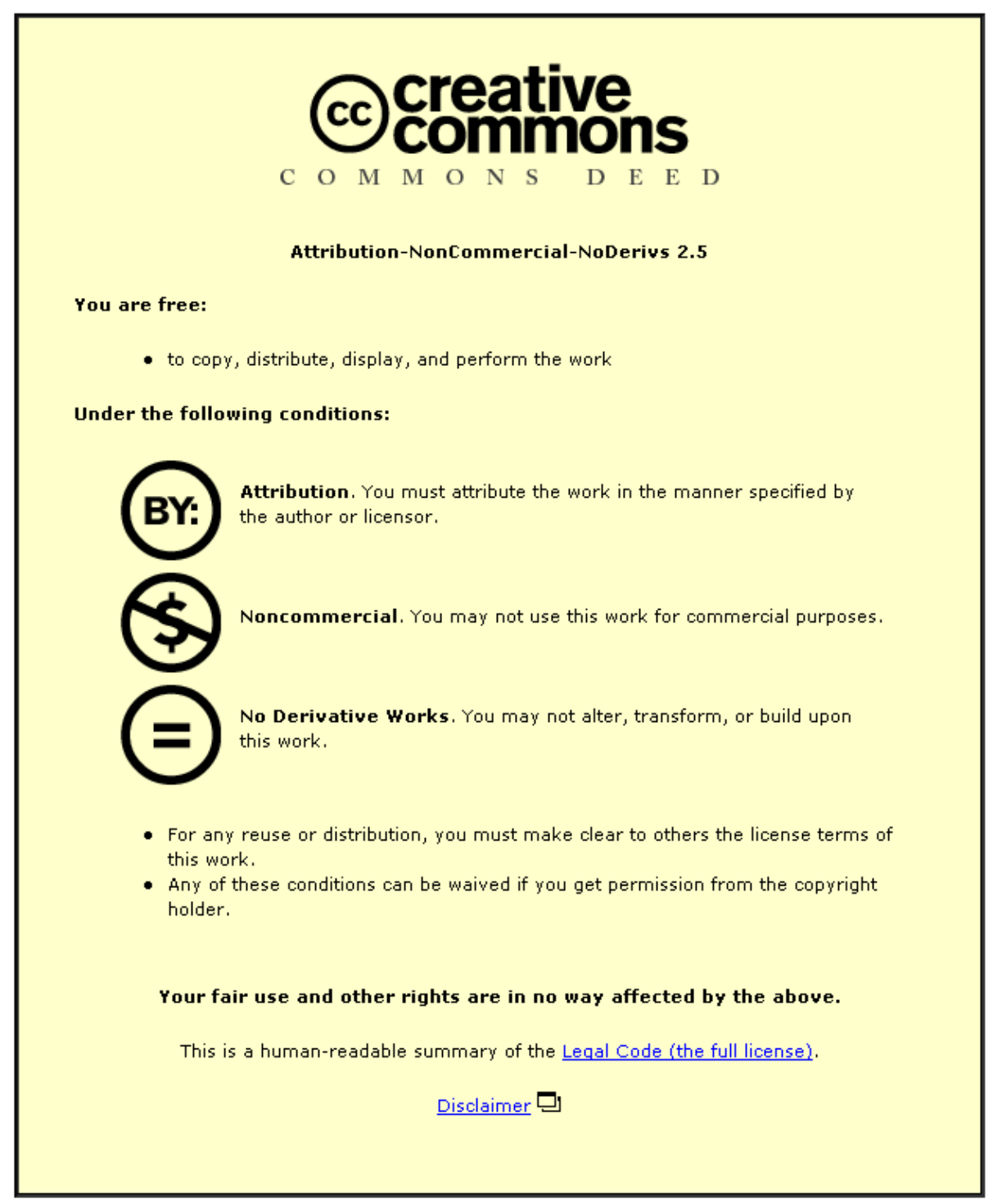

For the full text of this licence, please go to: http://creativecommons.org/licenses/by-nc-nd/2.5/ 


\title{
FROST HEAVE IN COLLOIDAL SOILS*
}

\author{
STEPHEN PEPPIN ${ }^{\dagger}$, APALA MAJUMDAR ${ }^{\dagger}$, ROBERT STYLE $^{\dagger}$, AND GRAHAM SANDER ${ }^{\ddagger}$
}

\begin{abstract}
We develop a mathematical model of frost heave in colloidal soils. The theory accounts for heave and consolidation while not requiring a frozen fringe assumption. Two solidification regimes occur: a compaction regime in which the soil consolidates to accommodate the ice lenses, and a heave regime during which liquid is sucked into the consolidated soil from an external reservoir, and the added volume causes the soil to heave. The ice fraction is found to vary inversely with the freezing velocity $V$, while the rate of heave is independent of $V$, consistent with field and laboratory observations.
\end{abstract}

Key words. frost heave, colloids, Lie-Bäcklund transformation

AMS subject classifications. 86A40, 86A99

DOI. $10.1137 / 100788197$

1. Introduction. Frost heave is a phenomenon in which the ground surface deforms and heaves under the action of freezing and thawing. The process leads to peculiar geological features (patterned ground) in cold regions of Earth as well as to challenging engineering problems [51]. In the early twentieth century it was discovered that frost heave is caused by the formation of repetitive layers of segregated ice in the soil, called ice lenses $[46,10]$. The most natural explanation for heave expansion of ice upon freezing - has surprisingly little effect. In fact, Taber [46] and Zhu et al. [54] showed that frost heave occurs in soils saturated with benzene and argon, materials which contract upon freezing. The heave occurs because the water which forms the ice lenses is drawn from other regions of the soil, and the frozen part comes to contain an excess mass of ice. The amount of heave is highly correlated with the volume of ice lenses formed $[46,51]$.

Mathematical models of frost heave tend to adopt the frozen fringe assumption $[28,29,15,23,41]$. A partially frozen region of soil is assumed to exist ahead of (i.e., at temperatures warmer than) the warmest ice lens. A combination of factors allows the partially frozen soil to segregate, revealing a new lens of pure ice [28, 41]. Remarkably, frost heave experiments on highly compressible laboratory soils such as colloidal silica found that there is no pore ice near the ice lenses $[6,7,48,49]$. These results confirm early observations of Beskow that in clays and fine silts the soil between ice lenses is soft and unfrozen $[5,30]$ but have yet to be explained theoretically.

In the present work we develop a continuum theory of frost heave that does not depend on the frozen fringe assumption. The model bears some similarity to mushy layer models of alloy solidification $[20,53]$ in that we treat the region containing segregated ice lenses as a continuum rather than attempt to track individual ice crystals. In section 2 we discuss the phase diagram of a colloidal soil composed of silica microspheres, distinguishing between segregational freezing (ice lenses) and

*Received by the editors March 10, 2010; accepted for publication (in revised form) June 26, 2011; published electronically September 22, 2011. This research was supported by the King Abdullah University of Science and Technology (KAUST) award KUK-C1-013-04.

http://www.siam.org/journals/siap/71-5/78819.html

†OCCAM, Mathematical Institute, University of Oxford, OX1 3LB Oxford, UK (peppin@maths. ox.ac.uk,majumdar@maths.ox.ac.uk, style@maths.ox.ac.uk).

${ }^{\ddagger}$ Department of Civil and Building Engineering, Loughborough University, LE11 3TU Leicestershire, UK (g.sander@lboro.ac.uk). 
interstitial freezing (pore ice). The frost heave model is presented in section 3, and in section 4 we present results and compare these with experiments. Some analytical solutions for the frost heave model are given in the appendix for completeness.

2. Segregational and interstitial freezing. During the solidification of watersaturated soils two distinct types of freezing processes occur [21]. Segregational freezing involves macroscopic regions of ice segregating from the particle matrix. In contrast, interstitial freezing occurs when ice enters the pores between particles. At relatively fast freezing rates the transition from segregational to interstitial freezing occurs via nonequilibrium particle trapping effects [47, 40]; i.e., the particles are engulfed by the rapidly moving ice. As noted by Jackson, Uhlmann, and Chalmers [21], in soils experiencing significant frost heave the freezing rates are typically slow enough that particle trapping does not occur. In this case the equilibrium phase diagram of a soil determines conditions under which the soil experiences segregational or interstitial freezing. Segregation freezing can occur either stably producing a single planar ice lens or, in the presence of constitutional supercooling, unstably yielding multiple ice lenses $[25,35,36,37,38]$. In the following we determine the phase diagram of a laboratory soil composed of spherical silica particles, used in the experiments of Watanabe and Mizoguchi [49] and Watanabe [50].

The segregation freezing temperature $T_{f}(\phi)$ (analogous to the liquidus temperature in alloys) is a function of the particle volume fraction $\phi$ and is given by the equation

$$
T_{f}(\phi)=T_{m}\left(1-\frac{\Pi(\phi)}{\rho_{l} L_{f}}\right)
$$

where $T_{m}$ is the freezing temperature of pure water, $\Pi(\phi)$ is the osmotic pressure of the particles, $\rho_{l}$ is the density of water, and $L_{f}$ is the latent heat of fusion $[27,35]$. Figure 2.1(a) shows measurements of $\Pi(\phi)$ for silica spheres of radius $R=0.5 \mu \mathrm{m}$ [18]. The solid line is a fit to the data using the equation [36]

$$
\Pi(\phi)=\frac{\phi k_{B} T_{m}}{v_{p}} \frac{1+\sum_{k=1}^{n} a_{k} \phi^{k}}{1-\phi / \phi_{p}},
$$

where $k_{B}$ is Boltzmann's constant, $v_{p}=\frac{4}{3} \pi R^{3}$ is the particle volume, $\phi_{p}=0.64$ is the
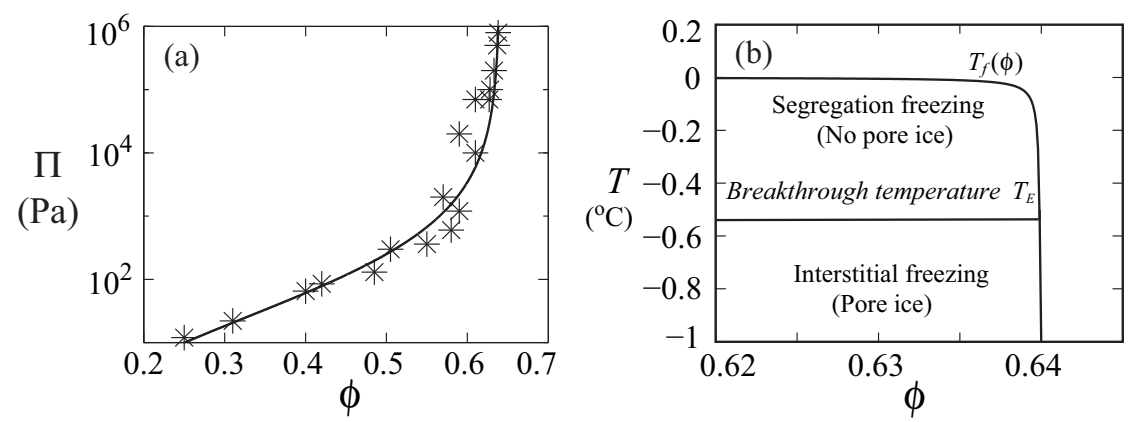

FIG. 2.1. (a) Measurements of the osmotic pressure of an aqueous suspension of silica spheres (radius $0.5 \mu \mathrm{m}$ ) [18], along with a fit to the data using $(2.2)$ with $a_{1}=5.5 \times 10^{3}, a_{2}=3.7 \times 10^{3}$, $a_{3}=1.3 \times 10^{5}, a_{4}=-8.7 \times 10^{4}$. (b) Phase diagram of an aqueous suspension of silica spheres, showing the freezing temperature $T_{f}(\phi)$ and the breakthrough temperature $T_{E}$. 
volume fraction at close packing, the $a_{k}$ are constants, and $n$ is chosen large enough to yield a good fit (usually $n=4$ is sufficient).

Figure 2.1(b) shows the liquidus temperature $T_{f}(\phi)$ calculated from (2.1). When the temperature is lowered below $T_{m}$, ice first forms outside of the soil pores as segregated ice. At a given temperature the concentration $\phi$ of the soil in equilibrium with pure ice is dictated by the $T_{f}(\phi)$ curve. Eventually the soil becomes fully consolidated $\left(\phi \rightarrow \phi_{p}\right)$, and a critical ice breakthrough temperature $T_{E}$ is reached $[7,44]$. At this temperature ice enters the pore space between particles, and interstitial freezing proceeds. The breakthrough temperature can be estimated from the pressure difference required for ice to enter the pore space. Thus, for close-packed spherical particles breakthrough occurs when $\Pi=\gamma / R_{p}$, where $\gamma$ is the ice-water surface energy and $R_{p}$ is the effective pore radius. For a hexagonally close-packed system with zero contact angle between the ice-water and water-particle interfaces, Hilden and Trumble [19] obtain $R_{p}=R / 11$. Inserting this expression for $\Pi$ into (2.1) gives

$$
T_{E}=T_{m}\left(1-\frac{11 \gamma}{\rho_{l} L_{f} R}\right) .
$$

Using typical values for ice $\left(\gamma=.03 \mathrm{~J} / \mathrm{m}^{2}, \rho_{l}=10^{3} \mathrm{~kg} / \mathrm{m}^{3}\right.$, and $\left.L_{f}=3.3 \times 10^{5} \mathrm{~J} / \mathrm{kg}\right)$ with $R=0.5 \mu \mathrm{m}$ gives $T_{E}=T_{m}-0.54{ }^{\circ} \mathrm{C}$ (Figure $2.1(\mathrm{~b})$ ).

The soil diffusivity $D(\phi)$ can be determined from the generalized Stokes-Einstein relation [42] as

$$
D(\phi)=\phi \frac{k(\phi)}{\eta} \frac{\partial \Pi(\phi)}{\partial \phi},
$$

where $k(\phi)$ is the permeability and $\eta$ the fluid viscosity. For $k$ we use the semiempirical expression [42]

$$
k(\phi)=\frac{2 R^{2}}{9 \phi}(1-\phi)^{b},
$$

where $b$ is a parameter accounting for viscous dissipation. Davis and Russel [12] have measured the permeability of silica microspheres, and their data can be fit well using
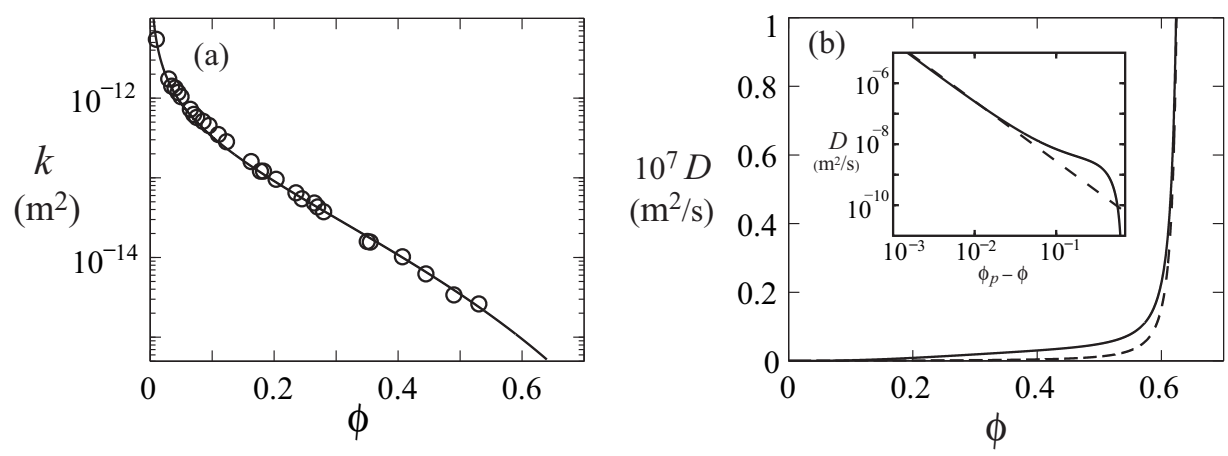

FIG. 2.2. (a) Measurements of the permeability $k(\phi)$ of a suspension of silica spheres [12] along with a fit of the data to the equation $k=\left(2 R^{2} / 9 \phi\right)(1-\phi)^{5}$. (b) Diffusivity of a suspension of silica spheres calculated using the Stokes-Einstein equation (2.4) (solid line) and the simpler equation (2.6) (dashed line). The inset shows the diffusivity plotted versus $\phi_{p}-\phi$. 
$b=5$, as shown in Figure 2.2(a). The diffusivity calculated from (2.4) is shown as the solid line in Figure 2.2(b).

In order to make the problem analytically tractable and simplify the numerics we consider in the remainder of this work a diffusivity of the form

$$
D(\phi)=\frac{D_{0}}{\left(1-\phi / \phi_{p}\right)^{2}}
$$

where $D_{0}=3.8 \times 10^{-11} \mathrm{~m}^{2} / \mathrm{s}$ is a constant. This expression, plotted as the dashed line in Figure 2.2(b), captures the essential limiting behaviors that $D$ approaches a constant in the dilute limit and diverges in the fully consolidated (close-packing) limit $[42,36]$. In [35] we worked in the low-concentration regime, where the diffusivity is approximately a constant. In this paper, we are interested in the close-packing limit, and, as can be seen from Figure 2.2(b), (2.6) shows good agreement with the exact expression (2.4) in the close-packing regime $\phi \rightarrow \phi_{p}$.

3. One-dimensional model. We develop a model of the unidirectional solidification system studied by Watanabe and Mizoguchi [49] and Watanabe [50]. In their experiments a cell containing water and silica of an initial particle fraction $\phi_{0}$ is pulled at a constant rate $V$ through a fixed temperature gradient $G_{T}$ (Figure 3.1). In the experiments the latent heat of solidification was transported through the glass sides of the solidification cell, and the temperature was assumed linear [49]. This is typically referred to as the "frozen temperature" approximation [13]. We consider a coordinate system fixed with respect to the isotherms and define the $z=0$ position as the $0^{\circ} \mathrm{C}$ isotherm. The temperature profile in the system is then given by the linear relation

$$
T(z)=T_{m}+G_{T} z .
$$

If $V$ is sufficiently small, a single planar ice lens is observed to push the particles ahead indefinitely $[6,49,37]$. At faster rates segregated ice forms in the interior of the particle layer yielding a mixed phase (mushy) region composed of ice lenses and unfrozen colloid $[6,37]$. We treat this mushy layer as a continuum characterized by an average segregated-ice volume fraction $g$, and so the overall particle fraction in this region is $(1-g) \phi$. The boundary between the mushy region and the unfrozen

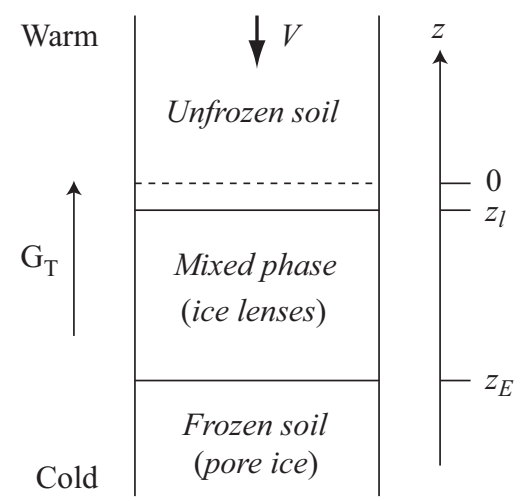

FIG. 3.1. Schematic diagram of the solidification cell used by Watanabe [50]. The sample is moved at a fixed rate $V$ through a constant temperature gradient $G_{T}$. Ice enters the pores at $z_{E}$, while $z_{l}$ demarcates the boundary between the mixed-phase region and unfrozen soil. 
silica soil is denoted by $z_{l}$. Similarly to mushy layer models of alloy solidification, we assume that the mixed phase region is locally in equilibrium [53], so that the particle concentration $\phi$ and the temperature $T$ are coupled by the phase diagram.

With the local equilibrium assumption, the temperature at $z_{l}$ is then $T_{l}=T_{f}\left(\phi_{l}\right)$, where $\phi_{l}=\phi\left(z_{l}\right)$. This assumption requires some comment, as it is not obvious that the mushy region can always be assumed to be locally in equilibrium. For example, depending on the rate of freezing the particles may not have time to maintain their equilibrium value of $\phi$ in response to changes in temperature and ice fraction. Here we assume that ice lens growth rates are always slow enough for local equilibrium to be maintained. This assumption is likely to be a good one at the slow freezing rates typical of frost heaving soils but may break down in some industrial settings involving rapid solidification [14].

From Figure 2.1(b) it is evident that for most of the range in $\phi$ the liquidus temperature $T_{f}(\phi)$ is very near $T_{m}$. Over this range we can make the approximation $T_{l}=T_{m}$. The position of the mixed phase boundary is then given by

$$
z_{l}=0 \text {. }
$$

Using (2.3) and (3.1) the breakthrough position $z_{E}$ at which ice enters the pores is obtained as

$$
z_{E}=-\frac{11 T_{m} \gamma}{\rho_{l} L_{f} R G_{T}}
$$

In order to predict heave, it is necessary to consider conservation of mass in the system. In the mushy region $0>z>z_{E}$, $\phi$ is coupled to the temperature $T$ by the phase diagram and is therefore known. We neglect all mass transport in the frozen region $z<z_{E}$ where the temperatures are below $T_{E}$. Thus we have only to find $\phi(z, t)$ in the region $z>0$. As mentioned in the introduction, the expansion of ice on freezing is not essential to the frost heave phenomenon, and we ignore it here. In the experiments of Watanabe and Mizoguchi [49] and Watanabe [50] the cell was oriented horizontally, and the effects of gravity were negligible. Therefore, the difference in density between the water and soil particles will also be ignored.

Given these approximations, in a frame of reference fixed with respect to the isotherms, conservation of mass in the region $z>0$ can be written as

$$
\frac{\partial \phi}{\partial t}=\frac{\partial}{\partial z}\left(D(\phi) \frac{\partial \phi}{\partial z}+V \phi\right)
$$

where $D(\phi)$ is given by $(2.6)$, with boundary conditions

$$
\begin{gathered}
g \phi V=-D(\phi) \frac{\partial \phi}{\partial z} \quad(z=0), \\
\phi \rightarrow 0 \quad(z \rightarrow \infty) .
\end{gathered}
$$

The first boundary condition (3.5) expresses conservation of mass at the mixed phase/ unfrozen-soil boundary. When the ice fraction $g$ is 1 , all the soil is pushed ahead by a single stable ice lens. When $g<1$, multiple ice lenses form, yielding a mixed phase region. The second boundary condition (3.6) ensures that a reservoir of pure water is available to keep the soil saturated. The initial condition is that of a layer of soil of height $L$ and concentration $\phi_{0}$, above which is the water reservoir:

$$
\phi(z, 0)= \begin{cases}\phi_{0}, & 0<z \leq L \\ 0, & z>L\end{cases}
$$


3.1. Marginal stability. Equations (3.4)-(3.7) contain the unknown ice fraction $g$. Here we neglect mass transport in the mushy region and treat $g$ as a constant. A similar assumption lead to good results in the case of alloy mushy layers [20]. This assumption, sometimes referred to as the Scheil approximation, requires the mass diffusivity $D$ to be much less than the thermal diffusivity $\kappa$. This approximation will be a good one at low particle concentrations but will eventually fail as $\phi \rightarrow \phi_{p}$ and $D$ diverges. Nevertheless, in real systems the particle diffusivity will increase rapidly but will not actually diverge to infinity owing to compressibility effects (divergent behavior occurs only for idealized "hard" spheres). In many systems of interest the particle diffusivity will remain smaller than the thermal diffusivity. Second, upon reaching close contact in the mushy region, the particles may bond to each other and to particles in the frozen part of the sample [45], inhibiting diffusive motion. While it would be interesting mathematically to explore more fully the effects of diffusion in the mushy region, we believe the Scheil approximation remains useful in colloidal systems and hope it will stimulate further modeling efforts.

To determine $g$ we use a marginal equilibrium condition [52] given by

$$
G_{T}=G_{T_{f}} \quad(z=0),
$$

where $G_{T_{f}}=\partial T_{f}(\phi) / \partial z$ is the liquidus temperature gradient at $z=0$. Equation (3.8) ensures that the system is everywhere in local equilibrium, including just ahead of the mushy region. Conservation of mass at the interface (3.5) along with (3.8), (2.4), and (2.1) implies that $g \phi V=-D \partial \phi / \partial z=\phi k \rho_{l} L_{f} G_{T} / \eta T_{m}$ or, equivalently,

$$
g=\frac{\rho_{l} L_{f} G_{T} k}{T_{m} \eta V} .
$$

Equation (3.9) can be used to determine the dependence of $g$ on the freezing velocity $V$. From the phase diagram in Figure 2.1 it is evident that the particle concentration $\phi$ in the mushy region will rapidly approach $\phi_{p}$ in $z<0$. If we assume that $\phi(z=0)=\phi_{p}$, then, for a given particle radius $R$, the permeability $k=k\left(\phi_{p}\right)$ is a constant (cf. (2.5)). In this case (3.9) shows that $g \sim V^{-1}$. This scaling is shown in Figure 3.2 , along with experimental data. The data can be fit to the expression

$$
g=\frac{V_{c}}{V}
$$

where $V_{c}=0.15 \mu \mathrm{m} / \mathrm{s}$ is a critical freezing rate above which the interface becomes unstable. Equation (3.9) however gives a much smaller critical velocity $V_{c}=\rho_{l} L_{f} G_{T} k / T_{m} \eta$

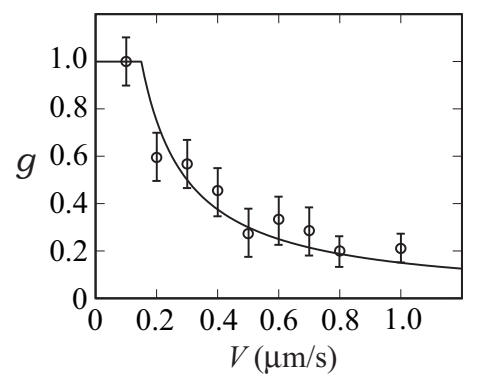

FIG. 3.2. Plot of the ice fraction $g$ versus the pulling speed $V$ measured by Watanabe [50]. The ice fraction is calculated as $g=l_{t h} /\left(l_{t h}+l_{s p}\right)$, where $l_{t h}$ and $l_{s p}$ are, respectively, the measured thicknesses of and spacings between the ice lenses. 
$\sim .01 \mu \mathrm{m} / \mathrm{s}$. One possible explanation for the discrepancy is the significant polydispersity in particle size used by Watanabe and Mizoguchi [49]. If the smallest particles rest against the ice lens during freezing [31,34], they will reduce the effective particle radius $R$ at $z=0$ and hence via (2.5) reduce the permeability. More importantly, the ice lenses will reject impurities such as dissolved air molecules and ions present in the water. As the water must pass such impurities to reach the ice, the effective permeability is reduced still further. Using $V_{c}=0.15 \mu \mathrm{m} / \mathrm{s}$ in (3.10) and (3.9) gives an average particle radius of $R \sim 0.1 \mu \mathrm{m}$, which seems not unreasonable. In order to fully resolve this issue it will be necessary to either perform experiments on highly monodisperse samples with no impurities or extend the theory to account for polydispersity and the presence of ionic solutes and dissolved air.

4. Results and discussion. We nondimensionalize the governing equations by introducing the variables $\hat{z}=z / L, \hat{t}=t D_{0} / L^{2}$, and $\Phi=\phi / \phi_{p}$ into (3.4)-(3.7) to give, upon dropping the carets,

$$
\begin{gathered}
\frac{\partial \Phi}{\partial t}=\frac{\partial}{\partial z}\left[\mathcal{D} \frac{\partial \Phi}{\partial z}+\operatorname{Pe} \Phi\right] \\
g \Phi P e+\mathcal{D} \frac{\partial \Phi}{\partial z}=0 \quad(z=0), \\
\Phi \rightarrow 0 \quad(z \rightarrow \infty), \\
\Phi(z, 0)= \begin{cases}\Phi_{0}, & 0<z \leq 1, \\
0, & z>1,\end{cases}
\end{gathered}
$$

where $\mathcal{D}=D / D_{0}=(1-\Phi)^{-2}, P e=V L / D_{0}$ is the Peclet number, and $\Phi_{0}=\phi_{0} / \phi_{p}$.

Solutions to (4.1)-(4.4) were obtained numerically using the method of lines [26]. We first consider the case $g=1$, in which a single ice lens forms at $\tilde{z}=0$ and pushes ahead the soil particles. In this case an analytical solution to the governing equations can be obtained via a Lie-Bäcklund transformation (see the appendix), providing a useful check on the numerics. Figure 4.1(a)-(e) shows results of the model for the case $L=4 \mathrm{~cm}, G_{T}=0.5^{\circ} \mathrm{C} / \mathrm{m}, \phi_{0}=0.5, V=0.15 \mu \mathrm{m} / \mathrm{s}$, and $g=1$ (from (3.10) with $V_{c}=0.15 \mu \mathrm{m} / \mathrm{s}$ ), corresponding to the dimensionless parameters $P e=160$ and $\Phi_{0}=0.78$. For clarity of presentation the results are plotted in the cell frame $\tilde{z}=z+V t$. Thus Figure 4.1(a) shows the initial state of the soil, while in figures (b)-(e) the temperature at the base $\tilde{z}=0$ is being lowered at the rate $G_{T} V$. Two distinct regimes of behavior occur.

In the consolidation regime, Figures 4.1(b) and (c), the soil consolidates as the ice forms. During this period the water which feeds the ice layer comes from the consolidated soil ahead of the ice-soil boundary, and no heave occurs. In reality we would expect a small amount of heave to occur owing to the volume expansion of ice. However, as noted in the introduction, volumetric expansion is not essential to frost heave and has been neglected in the model.

In the heaving regime, Figures 4.1(d) and (e), the soil is fully consolidated. During this period the ice grows by displacing (heaving) the entire column of soil. The water which feeds the ice layer in this case comes from the external reservoir. To quantify the amount of heave we define the upper surface of the soil as the highest point at which $\phi \geq \phi_{0}$. With this definition the height of the soil actually decreases in Figures 4.1(b) and (c). This is due to swelling, where the preconsolidated soil expands 

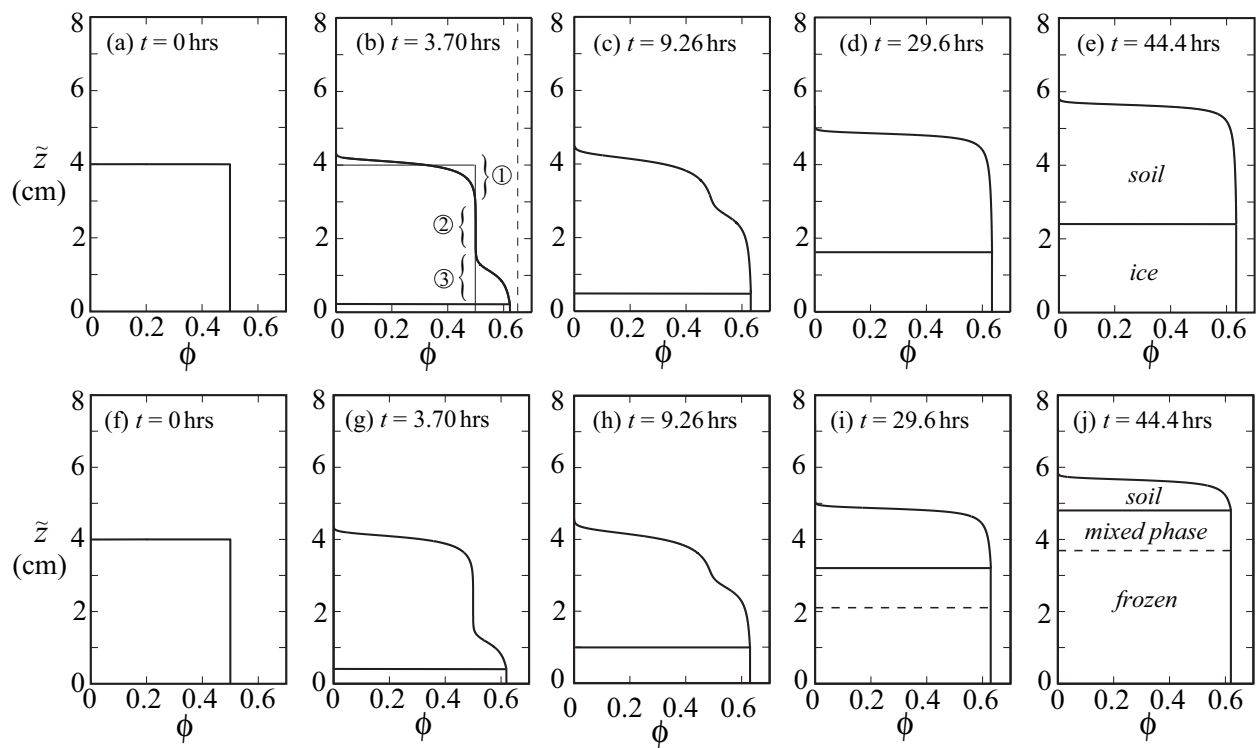

FIG. 4.1. Plots of $\phi(z, t)$ in the case $g=1$, (a)-(e), and $g=0.5$, (f)-(j). In (b)-(e) and (g)-(j) the horizontal solid line designates the $0^{\circ} \mathrm{C}$ isotherm, while the dashed line in (i) and (j) designates the breakthrough temperature $T_{E}$ isotherm.

upward into the water reservoir. To distinguish between swelling and heave, we have superimposed the initial undisturbed soil profile on Figure 4.1(b). The dashed line shows the maximum close-packing concentration $\phi_{p}$. At $t=3.70 \mathrm{hrs}$ three distinct regions can be discerned in the figure: (1) a swelling region where the upper surface of the soil has expanded into the water reservoir, (2) an undisturbed portion of soil, and (3) a consolidated soil layer above the ice interface. Frost heave commences only after Figure 4.1(c), when the consolidated layer has caught up with the surface of the soil. Thus the present model can simulate both swelling and heaving processes simultaneously.

In Figures 4.1(f)-(j) the freezing speed has been increased to $V=0.3 \mu \mathrm{m} / \mathrm{s}$, and all other parameter values are unchanged, leading to $P e=320$ and, from (3.10) with $V_{c}=0.15 \mu \mathrm{m} / \mathrm{s}, g=0.5$. At this higher freezing rate a mixed phase region forms containing both unfrozen soil and segregated ice. Figures 4.1(g) and (h) show the consolidation regime, while (i) and (j) illustrate the heaving regime. In (i) and (j) the dashed line shows the $T_{E}$ isotherm determined by (3.3); below the dashed line the soil between ice lenses is no longer unfrozen but contains pore ice. The $T_{E}$ isotherm was not shown in Figures 4.1(a)-(e) because in that case the soil is entirely pushed ahead by the ice and remains unfrozen. Only in the case $g<1$ does the soil move to lower temperatures allowing for ice breakthrough to occur. Note that if the soil were initially preconsolidated to the breakthrough pressure $11 \gamma / R$ (or larger values), ice would simply invade the pore space and no heave would occur. Thus $11 \gamma / R$ represents the maximum frost heave pressure.

The heaving of the soil is shown in Figure 4.2. During the consolidation phase we ignore the effects of swelling and set the heave rate to zero. During the heaving phase, the heave magnitude is obtained by tracking the highest point at which $\phi \geq \phi_{0}$ (i.e., the upper surface of the soil). After an initial transient the heave is a linear function 


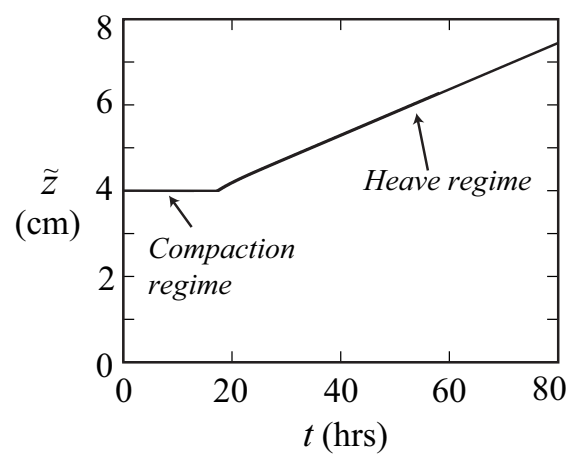

FIG. 4.2. Height of the soil as a function of time.

of time. Figure 4.2 shows the heave for both cases in Figure 4.1, $V=0.15 \mu \mathrm{m} / \mathrm{s}$ and $V=0.3 \mu \mathrm{m} / \mathrm{s}$, and the curves are almost indistinguishable. This result, that the heave rate is independent of the rate of freezing, is in agreement with field and laboratory observations of frost heave $[5,32,33]$. An explanation for this result can be obtained by writing the governing equations in a frame of reference fixed with respect to the soil/water interface (moving at the heaving rate $u$ ) as

$$
\begin{gathered}
\frac{\partial \phi}{\partial t}=\frac{\partial}{\partial z}\left(D(\phi) \frac{\partial \phi}{\partial z}+u \phi\right), \\
g \phi V=-D(\phi) \frac{\partial \phi}{\partial z} .
\end{gathered}
$$

At steady state $\partial \phi / \partial t=0$ so that (4.5) and (4.6) can be combined to give $g V=u$. Since $g \sim V^{-1}$ (see (3.10)), $u$ is independent of $V$.

This result implies that the heave rate of this system is determined by only two characteristic parameters: the soil permeability, $k$, and the temperature gradient in the soil, $G_{T}$. All the other parameters in the equation are soil-invariant. This can be related to the segregation potential concept of Konrad and Morgenstern [22, 24]. They showed that, for a freezing soil, the segregation potential $S P=u / G_{T}$ is approximately constant for a given soil. Our result gives theoretical support for the segregation potential concept in the case of colloidal soils with no frozen fringe.

5. Conclusions. In this paper we have investigated the heaving of a freezing colloidal soil. Experiments have shown that ice lenses can form in such systems without the presence of a frozen fringe. We have derived a model that accounts for this observation by predicting the heave rate of a soil when no frozen fringe is present. Our results show that there are typically two important regimes that occur during freezing. Initially, the freezing process causes excess liquid to be sucked out of the soil, compacting the soil particles together. During this consolidation regime, there is little heave. Once the soil is close-packed, liquid starts to be sucked through the soil from the external reservoir to the freezing front where it freezes. The extra volume brought into the soil causes the soil to expand, and thus the surface height of the soil will increase (the heaving regime). A key result is that the heave rate of the soil depends only on material parameters of the water, the close-packed permeability of the soil, and the temperature gradient and is independent of the speed of propagation of the freezing front. This is consistent with experiments and provides theoretical 
support for application of the segregation potential concept to colloidal soils with no frozen fringe. Finally, in the appendix we have provided exact and asymptotic solutions to our model in the case of the growth of a single ice lens. This is of use both as a convenient check of numerical simulations of frost heaving and in modeling the slow growth of a single ice lens.

\section{Appendix: Analytical results.}

6.1. Lie-Bäcklund solution. In the case $g=1$ a Lie-Bäcklund transformation [39] can be used to obtain an analytical solution for the system (4.1)-(4.4). This method is particularly useful for obtaining exact analytical solutions with nonlinear diffusivity and conductivity functions, and such solutions provide a demanding test of numerical simulations $[16,3,4]$. In what follows we set $g=1$ and derive the corresponding Lie-Bäcklund analytical solution. We note in passing that (4.1)-(4.4), in the case $g=1$, are employed by Davis, Russel, and Glantschnig [11] in their study of ultrafiltration, and hence this method yields a solution to that problem as well.

We introduce a change of variable and define

$$
\begin{aligned}
& \Phi^{*}\left(z^{*}(z, t), t\right)=\frac{1}{1-\Phi} \\
& z^{*}=\int_{0}^{z} 1-\Phi\left(z^{\prime}, t\right) d z^{\prime}
\end{aligned}
$$

Then

$$
\Phi=1-\frac{1}{\Phi^{*}}
$$

and

$$
\begin{gathered}
\frac{\partial z^{*}}{\partial z}=\frac{1}{\Phi^{*}\left(z^{*}, t\right)} \\
\frac{\partial z^{*}}{\partial t}=-\int_{0}^{z} \frac{\partial \Phi}{\partial t}\left(z^{\prime}, t\right) d z^{\prime}=-\frac{1}{\Phi^{*}} \Phi_{z^{*}}^{*}-\frac{P e}{\Phi^{*}}\left(\Phi^{*}-1\right) .
\end{gathered}
$$

We use the chain rule and the definitions (6.1) to obtain the following:

$$
\frac{\partial \Phi}{\partial t}=\frac{1}{\Phi^{* 2}}\left[\Phi_{z^{*}}^{*} \frac{\partial z^{*}}{\partial t}+\frac{\partial \Phi^{*}}{\partial t}\right]=-\frac{\left(\Phi_{z^{*}}^{*}\right)^{2}}{\left(\Phi^{*}\right)^{3}}-\frac{P e}{\left(\Phi^{*}\right)^{3}} \Phi_{z^{*}}^{*}\left(\Phi^{*}-1\right)+\frac{\Phi_{t}^{*}}{\left(\Phi^{*}\right)^{2}}
$$

and

$$
\frac{\partial}{\partial z}\left[\frac{1}{(1-\Phi)^{2}} \Phi_{z}+P e \Phi\right]=\frac{1}{\Phi^{*}} \frac{\partial}{\partial z^{*}}\left[\frac{\Phi_{z^{*}}^{*}}{\Phi^{*}}+P e \frac{1}{\Phi^{*}}\left(\Phi^{*}-1\right)\right]
$$

When we substitute (6.2) and (6.3) into (4.1), the governing partial differential equation for the transformed variable $\Phi^{*}$ becomes

$$
\frac{\partial \Phi^{*}}{\partial t}=\frac{\partial}{\partial z^{*}}\left[\Phi_{z^{*}}^{*}+\operatorname{Pe}\left(\Phi^{*}-1\right)\right]
$$

The boundary condition on $z=0$ can be transformed similarly, and the statement of 
the transformed problem is thus given by

$$
\begin{aligned}
& \frac{\partial \Phi^{*}}{\partial t}=\frac{\partial}{\partial z^{*}}\left[\Phi_{z^{*}}^{*}+P e\left(\Phi^{*}-1\right)\right] \\
& \Phi_{z^{*}}^{*}+P e\left(\Phi^{*}-1\right)=0 \quad \text { on } z^{*}=0 \\
& \Phi^{*} \rightarrow 1, \quad z^{*} \rightarrow \infty, \\
& \Phi^{*}\left(z^{*}, 0\right)= \begin{cases}\frac{1}{1-\Phi_{0}}, & 0<z^{*} \leq 1-\Phi_{0}, \\
1, & z^{*}>1-\Phi_{0}\end{cases}
\end{aligned}
$$

(since $\left.z=\int_{0}^{z^{*}} \Phi^{*}\left(z^{\prime}, t\right) d z^{\prime}\right)$.

The partial differential equation for $\Phi^{*}$ can be transformed into the heat equation using a second change of variable (see [9] for similar examples in the context of Richards's equation with arbitrary time-dependent surface fluxes) by choosing

$$
\begin{aligned}
& Z=P e z^{*}, \quad T=P e^{2} t, \\
& \bar{\phi}(Z, T)=\left(\Phi^{*}-1\right) \mathrm{e}^{\frac{Z}{2}+\frac{T}{4}} .
\end{aligned}
$$

Then the system (6.5) is reduced to the following initial-boundary value problem for the heat equation:

$$
\begin{aligned}
& \bar{\phi}_{T}=\bar{\phi}_{Z Z}, \\
& \bar{\phi}_{Z}+\frac{\bar{\phi}}{2}=0, \quad Z=0, \\
& \bar{\phi} \rightarrow 0, \quad Z \rightarrow \infty, \\
& \bar{\phi}(Z, 0)= \begin{cases}\frac{\Phi_{0}}{1-\Phi_{0}} \mathrm{e}^{\frac{Z}{2}}, & 0<Z \leq P e\left(1-\Phi_{0}\right), \\
0, & Z>P e\left(1-\Phi_{0}\right) .\end{cases}
\end{aligned}
$$

A semiexplicit solution of (6.7) can be found in [8], and the interested reader is referred there for technical details. This semiexplicit solution consists of two integral contributions, as follows:

$$
\begin{gathered}
\bar{\phi}(Z, T)=\frac{\Phi_{0}}{2\left(1-\Phi_{0}\right)} \int_{0}^{P e\left(1-\Phi_{0}\right)}\left\{\frac{1}{\sqrt{\pi T}}\left[\mathrm{e}^{-\left(Z-Z^{\prime}\right)^{2} / 4 T}+\mathrm{e}^{-\left(Z+Z^{\prime}\right)^{2} / 4 T}\right]\right. \\
\left.(6.8)+\mathrm{e}^{T / 4-\left(Z+Z^{\prime}\right) / 2} \operatorname{erfc}\left[\frac{Z+Z^{\prime}}{2 \sqrt{T}}-\frac{1}{2} \sqrt{T}\right]\right\} \mathrm{e}^{\frac{Z^{\prime}}{2}} d Z^{\prime},
\end{gathered}
$$

where erfc is the complementary error function defined in [1]. The original distribution $\phi(z, t)$ can be recovered from (6.8) using the identity

$$
\phi(z, t)=\phi_{p}\left[\frac{\bar{\phi}(Z, T)}{\mathrm{e}^{Z / 2+T / 4}+\bar{\phi}(Z, T)}\right]
$$

along with

$$
z=\frac{1}{P e} \int_{0}^{Z} \Phi^{*}\left(Z^{\prime}, T\right) d Z^{\prime}
$$

where $\Phi^{*}=1+\bar{\phi}(Z, T) \mathrm{e}^{-(Z / 2+T / 4)}$. The solution for $\phi(z, t)$ is therefore given parametrically through $\phi(Z, T), z(Z, T)$, and $t=T / P e^{2}$.

Copyright $@$ by SIAM. Unauthorized reproduction of this article is prohibited. 
6.2. Asymptotic results. The Lie-Bäcklund solution (6.8) yields in principle all information about the system, though to extract the relevant quantities it is still necessary to solve the integrals in (6.10) and (6.8). More explicit analytical results can be obtained by exploiting the asymptotic behavior evident in Figure 4.1. At early times there is a diffusive front at $z=-$ Pet. Before this front and the compaction front interact, a similarity solution can be obtained via the transformation $\eta=(z+$ $P e t) / \sqrt{t}$. Inserting this into (4.1) leads to the ordinary differential equation

$$
-\eta \frac{\mathrm{d} \Phi}{\mathrm{d} \eta}=2 \frac{\mathrm{d}}{\mathrm{d} \eta}\left[(1-\Phi)^{-2} \frac{\mathrm{d} \Phi}{\mathrm{d} \eta}\right]
$$

subject to $\Phi(-\infty)=\Phi_{0}$ and $\Phi(\infty)=0$.

Using methods similar to Fujita $[17,43]$ an exact solution to the above can be obtained as follows. Let

$$
x=(1-\Phi)^{-1}, \quad \psi=-\frac{\mathrm{d} \Phi}{\mathrm{d} \eta}, \quad \text { and } \quad w=\psi x^{3} .
$$

Combining these with (6.11) and manipulating the equations as in Sander, Norbury, and Weeks [43] gives

$$
\eta=2 x \frac{\mathrm{d} w}{\mathrm{~d} x}-2 w \quad \text { and } \quad\left(\frac{\mathrm{d} w}{\mathrm{~d} x}\right)^{2}=k-\ln w,
$$

where $k$ is a constant. Applying the boundary condition $\Phi \rightarrow 0$ as $\eta \rightarrow \infty$ and $w \rightarrow 0$ and integrating gives

$$
x=1+\int_{0}^{w} \frac{\mathrm{d} w}{\sqrt{k-\ln w}}
$$

with solution

$$
x=1+\sqrt{\pi} \mathrm{e}^{k} \operatorname{erfc}(\beta),
$$

where $\beta=\sqrt{k-\ln w}$. Since $w \rightarrow 0$ for both boundary conditions, $w$ has a maximum at $w_{m}=e^{k}$, and (6.15) gives $\Phi(\beta)$ for $0 \leq w \leq w_{m}$ or $0 \leq \beta<\infty$. From (6.13), $\eta(\beta)$ is given by

$$
\eta=2 \beta x-2 e^{k-\beta^{2}},
$$

covering the range $-2 e^{k} \leq \eta<\infty$. By combining (6.15) and (6.16), one branch of the solution for $\Phi(\eta)$ is obtained. The other branch occurs for $\eta<-2 e^{k}$, and by noting the symmetry in $w(x)$ around $w_{m}$, since $\left(w^{\prime}\right)^{2}=f(w)$, then integration of (6.13) yields

$$
x=1+\sqrt{\pi} e^{k}(2-\operatorname{erfc} \beta),
$$

along with

$$
\eta=-2 \beta x-2 e^{k-\beta^{2}}
$$

for $-\infty<\eta<-2 e^{k}$. The constant $k$ can be obtained from (6.17) using the boundary condition $x \rightarrow\left(1-\Phi_{0}\right)^{-1}$ as $\eta \rightarrow-\infty$ and $\beta \rightarrow \infty$ giving

$$
k=\ln \left(\frac{\Phi_{0}}{2 \sqrt{\pi}\left(1-\Phi_{0}\right)}\right) .
$$

Copyright $@$ by SIAM. Unauthorized reproduction of this article is prohibited. 
The compaction front can also be characterized asymptotically at early times. It moves forward and joins $\Phi=\Phi_{0}$ to the fully consolidated state $\Phi \approx 1$ at $z=0$. Take $\xi=\operatorname{Pe}(z-v P e t)$, where $V(v+1)$ is the speed of the compaction front and $v$ is a constant. Then with the similarity assumption $\Phi=\Phi(\xi)$ equation (4.1) becomes $-(v+1) \Phi_{\xi}=\left(\mathcal{D} \Phi_{\xi}\right)_{\xi}$, which can be integrated to obtain

$$
-(v+1) \xi=\int_{\Phi_{s}}^{\Phi} \frac{\mathcal{D}}{\left(\Phi-\Phi_{0}\right)} \mathrm{d} \Phi
$$

with solution

$$
\xi=\frac{1}{\left(1-\Phi_{0}\right)} \ln \frac{\left(\Phi_{s}-\Phi_{0}\right)(1-\Phi)}{\left(\Phi-\Phi_{0}\right)\left(1-\Phi_{0}\right)}-\left(\frac{1}{1-\Phi}-\frac{1}{1-\Phi_{s}}\right)
$$

where $\Phi_{s}=\Phi(z=0)$.

Assuming a sharp compaction front conservation of mass implies

$$
V v\left(1-\Phi_{0}\right)=V \Phi_{0}
$$

giving $v+1=\left(1-\Phi_{0}\right)^{-1}$. Global mass conservation then determines $\Phi_{s}$ according to

$$
\int_{\Phi_{s}}^{\Phi_{0}} \mathcal{D} \mathrm{d} \Phi=-(v+1) \int_{0}^{\infty}\left(\Phi-\Phi_{0}\right) \mathrm{d} \xi=-(v+1) \Phi_{0} P e^{2} t
$$

which gives

$$
\Phi_{s}=1-\frac{1-\Phi_{0}}{1+P e^{2} \Phi_{0} t}
$$

A sharp compaction front occurs, for example, when the colloid is composed of relatively large particles $(>1 \mu \mathrm{m})$ that experience little Brownian motion (for such particles the dilute diffusivity $D_{0}$ becomes small). This is illustrated in Figure 6.1, which shows the effect of decreasing $D_{0}$ (i.e., increasing the Peclet number $P e=$ $\left.V L / D_{0}\right)$ on the volume fraction profile in Figure 4.1(b). As $P e$ increases the front sharpens, forming a shock similar to that in Auzerais, Jackson, and Russel [2], and the asymptotic solution (6.20) shown as the dashed line becomes more accurate.
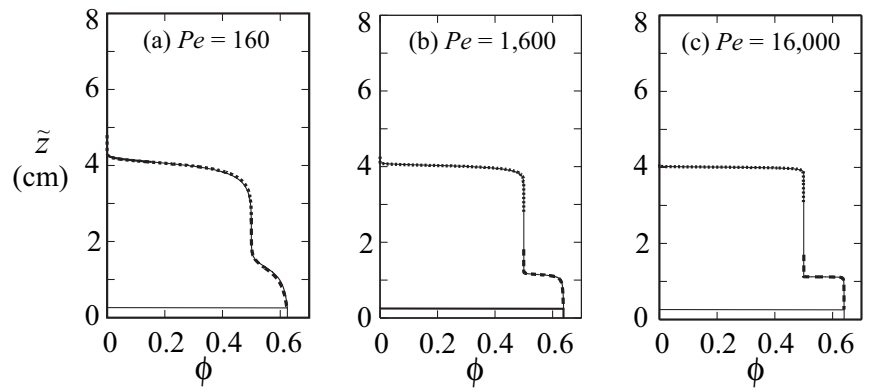

FIG. 6.1. Front-sharpening effect of decreasing $D_{0}$ (increasing Pe) on the profile in Figure 4.1(b). The dotted and dashed lines show the asymptotic solutions for the diffuse and compaction fronts, respectively.

Copyright $\odot$ by SIAM. Unauthorized reproduction of this article is prohibited. 
Acknowledgment. We thank the anonymous referees, whose comments and suggestions led to significant improvements to the manuscript.

\section{REFERENCES}

[1] M. Abramowitz and I. A. Stegun, Handbook of Mathematical Functions with Formulas, Graphs, and Mathematical Tables, Dover, New York, 1972.

[2] F. M. Auzerais, R. Jackson, And W. B. Russel, The resolution of shocks and the effects of compressible sediments in transient settling, J. Fluid Mech., 195 (1988), pp. 437-462.

[3] D. A. BARRY AND G. C. SANDER, Exact solutions for water infiltration with an arbitrary surface flux or nonlinear solute adsorption, Water Resour. Res., 27 (1991), pp. 2667-2680.

[4] D. A. Barry and G. Sposito, Analytical solution of a convection-dispersion model with timedependent transport coefficients, Water Resour. Res., 25 (1989), pp. 2407-2416.

[5] G. BEsKow, Soil freezing and frost heaving with special attention to roads and railroads, in The Swedish Geological Society, C, no. 375, Year Book no. 3, Technological Institute, Northwestern University, reprinted in Historical Perspectives in Frost Heave Research, P. B. Black and M. J. Hardenberg, eds., CRREL Special Report 91-23, 1991, pp. 37-157.

[6] S. C. Brown, Soil Freezing, Ph.D. thesis, University of Reading, Reading, UK, 1984.

[7] S. C. Brown And D. PaYne, Frost action in clay soils. II. Ice and water location and suction of unfrozen water in clays below $0^{\circ} \mathrm{C}$, J. Soil Sci., 41 (1990), pp. 547-561.

[8] H. S. Carslaw and J. C. Jaeger, Conduction of Heat in Solids, 2nd ed., Oxford University Press, Oxford, UK, 1986.

[9] J.-M. Chen, Y.-C. Tan, C.-H. Chen, and J. Y. Parlange, Analytical solutions for linearized Richards equation with arbitrary time-dependent surface fluxes, Water Resour. Res., 37 (2001), pp. 1091-1093.

[10] J. G. Dash, A. W. Rempel, and J. S. Wettlaufer, The physics of premelted ice and its geophysical consequences, Rev. Mod. Phys., 78 (2006), pp. 695-741.

[11] K. E. Davis, W. B. Russel, and W. J. Glantschnig, Settling suspensions of colloidal silica: Observations and X-ray measurements, J. Chem. Soc. Faraday Trans., 87 (1991), pp. 411424.

[12] K. E. Davis And W. B. Russel, An asymptotic description of transient settling and ultrafiltration of colloidal dispersions, Phys. Fluids A, 1 (1989), pp. 82-100.

[13] S. H. Davis, Theory of Solidification, Cambridge University Press, Cambridge, UK, 2001.

[14] S. Deville, E. Maire, G. Bernard-Granger, A. Lassalle, A. Bogner, C. Gauthier, J. Leloup, AND C. Guizard, Metastable and unstable cellular solidification of colloidal suspensions, Nature Mat., 8 (2009), pp. 966-972.

[15] A. C. Fowler, Secondary frost heave in freezing soils, SIAM J. Appl. Math., 49 (1989), pp. 991-1008.

[16] A. S. Fokas And Y. C. Yortsos, On the exactly solvable equation $S_{t}=\left[(\beta S+\gamma)^{-2} S_{x}\right]_{x}+$ $\alpha(\beta S+\gamma)^{-2} S_{x}$ occurring in two-phase flow in porous media, SIAM J. Appl. Math., 42 (1982), pp. 318-332.

[17] H. FuJITA, The exact pattern of a concentration-dependent diffusion in a semi-infinite medium, Part I, Textile Res. J., 22 (1952), pp. 757-760.

[18] J. J. Guo And J. A. Lewis, Aggregation effects on the compressive flow properties and drying behavior of colloidal silica suspensions, J. Am. Ceram. Soc., 82 (1999), pp. 2345-2358.

[19] J. L. Hilden and K. P. Trumble, Numerical analysis of capillarity in packed spheres: Planar hexagonal-packed spheres, J. Colloid Interface Sci., 267 (2003), pp. 463-474.

[20] H. E. Huppert And M. G. Worster, Dynamic solidification of a binary melt, Nature, 314 (1985), pp. 703-707.

[21] K. A. Jackson, D. R. Uhlmann, and B. Chalmers, Frost heave in soils, J. Appl. Phys., 37 (1966), pp. 848-852.

[22] J.-M. Konrad and N. R. Morgenstern, The segregation potential of a freezing soil, Can. Geotech. J., 18 (1981), pp. 482-491.

[23] J.-M. Konrad and C. Duquennoi, A model for water transport and ice lensing in freezing soils, Water Resour. Res., 29 (1993), pp. 3109-3124.

[24] J.-M. KonRaD, Frost susceptibility related to soil index properties, Can. Geotech. J., 36 (1999), pp. 403-417.

[25] J. S. LANGER, Instabilities and pattern formation in crystal growth, Rev. Mod. Phys., 52 (1980), pp. $1-28$. 
[26] H. S. Lee, C. J. Matthews, R. D. Braddock, G. C. Sander, and F. Gandola, A MatlaB method of lines template for transport equations, Environ. Modell. Softw., 19 (2004), pp. 603-614.

[27] P. F. Low, D. M. Anderson, And P. Hoekstra, Some thermodynamic relationships for soils at or below the freezing point. 1. Freezing point depression and heat capacity, Water Resour. Res., 4 (1968), pp. 379-394.

[28] R. D. Miller, Frost heaving in non-colloidal soils, in Proceedings of the 3rd International Conference on Permafrost, National Research Council of Canada, Edmonton, Alberta, 1978, pp. 708-713.

[29] K. O'Neill AND R. D. Miller, Exploration of a rigid ice model of frost heave, Water Resour. Res., 21 (1985), pp. 281-296.

[30] E. Penner, Frost heaving in soils, in Proceedings of the International Conference on Permafrost, National Research Council, Lafayette, IN, 1963, pp. 197-202.

[31] E. Penner, Pressure developed during the unidirectional freezing of water saturated porous materials, in Proceedings of the International Conference on Low Temperature Science, Vol. 1, Sapporo, Japan, 1966, pp. 1401-1412.

[32] E. Penner, Influence of freezing rate on frost heaving, Highway Res. Rec., 393 (1972), pp. 56-64.

[33] E. Penner, Aspects of ice lens growth in soils, Cold Reg. Sci. Tech., 13 (1986), pp. 91-100.

[34] E. PENner, Experimental pressure studies of frost heave mechanisms and the growth-fusion behavior of ice, in Proceedings of the Second International Conference on Permafrost, National Research Council, Yakutsk, USSR, 1973, pp. 377-384.

[35] S. S. L. Peppin, A. Majumdar, and J. S. Wettlaufer, Morphological instability of a nonequilibrium ice-colloid interface, Proc. Roy. Soc. Lond. A, 466 (2010), pp. 177-194.

[36] S. S. L. Peppin, J. A. W. Elliott, and M. G. Worster, Solidification of colloidal suspensions, J. Fluid Mech., 554 (2006), pp. 147-166.

[37] S. S. L. Peppin, J. S. Wettlaufer, and M. G. Worster, Experimental verification of morphological instability in freezing aqueous colloidal suspensions, Phys. Rev. Lett., 100 (2008), 238301.

[38] S. S. L. Peppin, M. G. Worster, and J. S. Wettlaufer, Morphological instability in freezing colloidal suspensions, Proc. Roy. Soc. Lond. A, 463 (2007), pp. 723-733.

[39] C. Rogers, M. P. Stallybrass, and D. L. Clements, On 2 phase filtration under gravity and with boundary infiltration-application of a Bäcklund transformation, Nonlinear Anal., 7 (1983), pp. 785-799.

[40] A. W. Rempel And M. G. Worster, The interaction between a particle and an advancing solidification front, J. Cryst. Growth, 205 (1999), pp. 427-440.

[41] A. W. Rempel, J. S. Wettlaufer, and M. G. Worster, Premelting dynamics in a continuum model of frost heave, J. Fluid Mech., 498 (2004), pp. 227-244.

[42] W. B. Russel, D. A. Saville, and W. R. Schowalter, Colloidal Dispersions, Cambridge University Press, Cambridge, UK, 1989.

[43] G. C. Sander, J. Norbury, AND S. W. Weeks, An exact solution to the nonlinear diffusionconvection equation for two-phase flow, Quart. J. Mech. Appl. Math., 46 (1993), pp. 709727.

[44] N. O. Shanti, K. Araki, and J. W. Halloran, Particle redistribution during dendritic solidification of particle suspensions, J. Am. Ceram. Soc., 89 (2006), pp. 2444-2447.

[45] M. Spannuth, S. G. J. Mockrie, S. S. L. Peppin, and J. S. Wettlaufer, Particle-scale structure in frozen colloidal suspensions from small-angle $x$-ray scattering, Phys. Rev. E, 83 (2011), 021402.

[46] S. TABER, Frost heaving, J. Geol., 34 (1929), pp. 428-461.

[47] D. R. Uhlmann, B. Chalmers, and K. A. Jackson, Interaction between particles and a solid-liquid interface, J. Appl. Phys., 35 (1964), pp. 2986-2993.

[48] K. Watanabe, M. Mizoguchi, T. Ishizaki, and M. Fukuda, Experimental study on microstructure near freezing front during soil freezing, in Proceedings of the International Symposium of Ground Freezing, Lulea, Sweden, 1997, pp. 187-194.

[49] K. Watanabe And M. Mizoguchi, Ice configuration near a growing ice lens in a freezing porous medium consisting of micro glass particles, J. Cryst. Growth, 213 (2000), pp. 135-140.

[50] K. WatanaBe, Relationship between growth rate and supercooling in the formation of ice lenses in a glass powder, J. Cryst. Growth, 237-239 (2002), pp. 2194-2198.

[51] P. J. Williams and M. W. Smith, The Frozen Earth: Fundamentals of Geocryology, Cambridge University Press, Cambridge, UK, 1989.

Copyright $@$ by SIAM. Unauthorized reproduction of this article is prohibited. 
[52] M. G. Worster, Solidification of an alloy from a cooled boundary, J. Fluid Mech., 167 (1986), pp. $481-501$.

[53] M. G. Worster, Solidification of fluids, in Perspectives in Fluid Dynamics, G. K. Batchelor, H. K. Moffatt, and M. G. Worster, eds., Cambridge University Press, Cambridge, UK, 2000, pp. 393-446.

[54] D.-M. Zhu, O. F. Vilches, J. G. Dash, B. Sing, and J. S. Wettlaufer, Frost heave in argon, Phys. Rev. Lett., 85 (2000), pp. 4908-4912.

Copyright (c) by SIAM. Unauthorized reproduction of this article is prohibited. 